\title{
Approaching the Lambertian limit in randomly textured thin-film solar cells
}

\author{
Stephan Fahr, ${ }^{1, *}$ Thomas Kirchartz, ${ }^{2}$ Carsten Rockstuhl, ${ }^{1}$ and \\ Falk Lederer ${ }^{1}$ \\ ${ }^{1}$ Friedrich-Schiller Universität Jena, Institute of Condensed Matter Theory and Solid State \\ Optics and Abbe Center of Photonics, Max-Wien-Platz 1, 07743 Jena, Germany \\ ${ }^{2}$ Imperial College London, Blackett Laboratory of Physics, South Kensington, London SW7 \\ 2AZ, UK \\ *stephan.fahr@uni-jena.de
}

\begin{abstract}
The Lambertian limit for solar cells is a benchmark for evaluating their efficiency. It has been shown that the performance of either extremely thick or extremely thin solar cells can be driven close to this limit by using an appropriate photon management. Here we show that this is likewise possible for realistic, practically relevant thin-film solar cells based on amorphous silicon. Most importantly, we achieve this goal by relying on random textures already incorporated into state-of-the-art superstrates; with the only subtlety that their topology has to be downscaled to typical feature sizes of about $100 \mathrm{~nm}$.
\end{abstract}

(C) 2011 Optical Society of America

OCIS codes: (040.5350) Photovoltaic; (290.0290) Scattering; (310.0310) Thin films; (350.6050) Solar energy.

\section{References and links}

1. R. Könenkamp, S. Muramatsu, H. Itoh, S. Matsubara, and T. Shimada, "Mobility-lifetime product in hydrogenated amorphous silicon,” Jpn. J. Appl. Phys. 29, L2155-L2158 (1990).

2. H. Okamoto, H. Kida, S. Nonomura, K. Fukumoto, and Y. Hamakawa, "Mobility-lifetime product and interface property in amorphous silicon solar cells," J. Appl. Phys. 54, 3236-3243 (1983).

3. D. L. Staebler and C. R. Wronski, "Reversible conductivity changes in discharge-produced amorphous Si," Appl. Phys. Lett. 31, 292-294 (1977).

4. J. Müller, B. Rech, J. Springer, and M. Vanecek, "TCO and light trapping in silicon thin film solar cells," Sol. Energy 77, 917-930 (2004).

5. M. Kroll, S. Fahr, C. Helgert, C. Rockstuhl, F. Lederer, and T. Pertsch, "Employing dielectric diffractive structures in solar cells - a numerical study," Phys. Status Solidi A 205, 2777-2795 (2008).

6. J. Grandidier, D. M. Callahan, N. Munday, and H. A. Atwater, "Light Absorption Enhancement in Thin-Film Solar Cells Using Whispering Gallery Modes in Dielectric Nanospheres," Adv. Mater. 23, 5 (2011).

7. C. Ulbrich, S. Fahr, J. Üpping, M. Peters, T. Kirchartz, C. Rockstuhl, R. Wehrspohn, A. Gombert, F. Lederer, and U. Rau, "Directional selectivity and ultra-light-trapping in solar cells," Phys. Status Solidi A 205, 2831-2843 (2008).

8. L. Cao, P. Fan, A. P. Vasudev, J. S. White, Z. Yu, W. Cai, J. A. Schuller, S. Fan, and M. L. Brongersma, "Semiconductor nanowire optical antenna solar absorbers," Nano Letters 10, 439-445 (2010).

9. J. Zhu, Z. Yu, G. F. Burkhard, C. Hsu, S. T. Connor, Y. Xu, Q. Wang, M. McGehee, S. Fan, and Y. Cui, "Optical absorption enhancement in amorphous silicon nanowire and nanocone arrays," Nano Lett. 9, 279-282 (2009).

10. C. Rockstuhl, S. Fahr, and F. Lederer, "Absorption enhancement in solar cells by localized plasmon polaritons," J. Appl. Phys. 104, 123102 (2008).

11. Y. A. Akimov, W. S. Koh, and K. Ostrikov, "Enhancement of optical absorption in thin-film solar cells through the excitation of higher-order nanoparticle plasmon modes," Opt. Express 17, 10195-10205 (2009).

12. E. Yablonovitch, "Statistical ray optics," J. Opt. Soc. Am. (1917-1983) 72, 899-907 (1982).

13. P. Campbell, "Enhancement of light absorption from randomizing and geometric textures," J. Opt. Soc. Am. B 10, 2410-2415 (1993).

\#146245 - \$15.00 USD Received 19 Apr 2011; revised 19 May 2011; accepted 23 May 2011; published 22 Jun 2011

(C) 2011 OSA

4 July 2011 / Vol. 19, No. S4 / OPTICS EXPRESS A865 
14. P. Campbell and M. A. Green, "Light trapping properties of pyramidally textured surfaces," J. Appl. Phys. 62, 243-249 (1987).

15. S. E. Han and G. Chen, "Toward the Lambertian limit of light trapping in thin nanostructured silicon solar cells," Nano Lett. 10, 4692-4696 (2010).

16. M. A. Green, "Enhanced evanescent mode light trapping in organic solar cells and other low index optoelectronic devices," Prog. Photovoltaics 19(4), 473-477 (2010).

17. Z. Yu, A. Raman, and S. Fan, "Fundamental limit of nanophotonic light trapping in solar cells," Proc. Natl. Acad. Sci. U.S.A. 107, 17491-17496 (2010).

18. O. Kluth, B. Rech, L. Houben, S. Wieder, G. Schöpe, C. Beneking, H. Wagner, A. Löffl, and H. W. Schock, "Texture etched $\mathrm{ZnO}: \mathrm{Al}$ coated glass substrates for silicon based thin film solar cells," Thin Solid Films 351, 247-253 (1999).

19. S. Nicolay, M. Despeisse, F. J. Haug, and B. Ballif, "Control of LPCVD ZnO growth modes for improved light trapping in thin film silicon solar cells," Sol. Energy Mater. Sol. Cells 95, 1031-1034 (2011).

20. M. G. Moharam, E. B. Grann, D. A. Pommet, and T. K. Gaylord, "Formulation for stable and efficient implementation of the rigorous coupled-wave analysis of binary gratings," J. Opt. Soc. Am. A 12, 1068-1076 (1995).

21. O. Vetterl, F. Finger, R. Carius, P. Hapke, L. Houben, O. Kluth, A. Lambertz, A. Mück, B. Rech, and H. Wagner, "Intrinsic microcrystalline silicon: a new material for photovoltaics," Sol. Energy Mater. Sol. Cells 62, 97-108 (2000).

22. M. Zeman, R. A. C. M. M. van Swaaij, J. W. Metselaar, and R. E. I. Schropp, "Optical modeling of a-Si:H solar cells with rough interfaces: effect of back contact and interface roughness," J. Appl. Phys. 88, 6436-6443 (2000).

23. C. Rockstuhl, S. Fahr, K. Bittkau, T. Beckers, R. Carius, F.-J. Haug, T. Söderström, C. Ballif, and F. Lederer, "Comparison and optimization of randomly textured surfaces in thin-film solar cells," Opt. Express 18, A335A341 (2010).

24. J. Steinhauser, S. Faÿ, N. Oliveira, E. Vallat-Sauvain, and C. Ballif, "Transition between grain boundary and intragrain scattering transport mechanisms in boron-doped zinc oxide thin films," Appl. Phys. Lett. 90, 142107 (2007).

25. M. Berginski, J. Hüpkes, M. Schulte, G. Schöpe, H. Stiebig, B. Rech, and M. Wuttig, “The effect of front ZnO:Al surface texture and optical transparency on efficient light trapping in silicon thin-film solar cells," J. Appl. Phys. 101, 074903 (2007)

26. W. H. Southwell, "Gradient-index antireflection coatings," Opt. Lett. 8, 584-586 (1983).

27. M. J. Keevers, T. L. Young, U. Schubert, and M. A. Green, "10\% Efficient CSG minimodules," Proceedings of the 22nd European Photovoltaic Solar Energy Conference and Exhibition, Milan (2007).

28. K. Bittkau, R. Carius, and C. Lienau, "Guided optical modes in randomly textured $\mathrm{ZnO}$ thin films imaged by near-field scanning optical microscopy," Phys. Rev. B 76, 035330 (2007).

29. C. Rockstuhl, F. Lederer, K. Bittkau, and R. Carius, "Light localization at randomly textured surfaces for solarcell applications," Appl. Phys. Lett. 91, 171104 (2007).

30. ASTM Standard G173-03, URL: http://www . astm.org

31. M. A. Green, "Lambertian light trapping in textured solar cells and light-emitting diodes: analytical solutions," Prog. Photovoltaics 10, 235-241 (2002).

32. C. Battaglia, J. Escarré, K. Söderström, L. Erni, L. Ding, G. Bugnon, A. Billet, M. Boccard, L. Barraud, S. de Wolf, F. Haug, M. Despeisse, and C. Ballif, "Nanoimprint lithography for high-efficiency thin-film silicon solar cells," Nano Lett. 11, 661-665 (2011).

\section{Introduction}

Thin-film solar cells are considered to be attractive candidates for photovoltaic elements. They can be fabricated at low costs on large areas. Due to the small thickness, the requirements imposed on the electronic properties of the involved materials are less severe than for the comparably thick wafer based cells made of crystalline silicon. Therefore, thin-film cells require less chemically pure material with less perfect structural order; which helps to reduce their fabrication costs. Among various possible inorganic materials suitable for thin-film solar cells, hydrogenated amorphous silicon $(\mathrm{aSi}: \mathrm{H})$ is potentially the most attractive material because it is abundant and nontoxic.

However, carrier collection in a-Si:H is hampered by their low initial mobility-lifetime product $[1,2]$ which is further reduced due to light induced degradation [3]. Consequently, the optimal thickness of the aSi:H thin-film solar cells is limited to $\approx 250 \mathrm{~nm}$. Although a further thickness increase leads to a higher absorption, insufficient carrier collection results in a reduction of the photocurrent and the fill factor. It is detrimental for the device performance that this

\#146245 - \$15.00 USD Received 19 Apr 2011; revised 19 May 2011; accepted 23 May 2011; published 22 Jun 2011

(C) 2011 OSA

4 July 2011 / Vol. 19, No. S4 / OPTICS EXPRESS A866 
thickness limitation yields to an insufficient light absorption of spectral components near the absorption edge [4]. This constitutes a, if not to say the major, limitation to be lifted. For that purpose a mere improvement of the material is out of the scope since it requires to abandon the material advantages of aSi:H. Instead, the absorptance has to be enhanced at wavelengths, where the absorption length is much larger than the absorber thickness. This can be achieved by integrating an assisting light trapping structure whose purpose is to steer the mould of light at the nanoscale inside the solar cell.

In order to increase the absorption of impinging sun light in aSi:H thin-films, various experimental schemes attracted considerable research interest recently. Some of them are rather explorative such as, e.g. the inclusion of microspheres [5,6], ultra light trapping schemes [7], nanowires $[8,9]$, or plasmonic nanostructures $[10,11]$. Currently, it is unclear whether at all, how, and also when such structures will find their way into commercial solar cells, since because of their higher costs they are required to perform appreciably better than structures already in place. To date the most common approach for an efficient light trapping in aSi:H is the use of textured surfaces made of a transparent conductive oxide (TCO). The purpose of these textures is to reduce reflection losses and to enhance the scattering of light, which leads to an effectively enhanced optical path length in the solar cell. For a Lambertian scattering texture, it was shown that the maximum achievable path length enhancement in the weakly absorbing limit amounts to $4 n^{2}$, with $n$ being the refractive index of the absorbing material [12]. This Lambertian limit, also called Yablonovitch limit, constitutes a referential benchmark for every possible light trapping scheme. Moreover, the race is on to propose structures that potentially even beat that limit, or operate at least as close as possible to it. For absorber thicknesses in the order of several hundreds of $\mu \mathrm{m}$ (being essentially wafer based solar cells), suitably tailored V-shaped grooves outperform the Lambertian limit $[13,14]$. Recently, deterministic asymmetric pyramids were equally found to provide a better performance than the Lambertian limit for thicknesses of the absorbing layer between 1 and $10 \mu \mathrm{m}$ [15]. On the other hand for extremely thin solar cells with a thickness in the order of only a few nanometers, the exploitation of a large number of diffraction channels also made it possible to reach the Lambertian limit and to even go beyond for the scenario, where an absorber is coupled to a scatterer, whose refractive index is larger than the index of the absorber $[16,17]$.

However, from a practical point of view it is difficult to find a sufficiently transparent scatterer with a refractive index much larger than 3.5, which would be required for silicon based thin-film solar cells. Furthermore, it is of utmost importance to reach this limit for thin-film solar cells with relevant thicknesses, i.e. thicknesses in the order of about $250 \mathrm{~nm}$. The aim of this Letter is to show that this can be achieved. This is a key step towards an ultimate implementation in a commercial device because it constitutes only a slight modification of the current fabrication technology. Therefore, the starting point for our consideration is a textured surface as already used in state-of-the-art thin-film solar cells made of $\mathrm{ZnO}$. These materials can be sputtered onto the superstrate and afterwards etched [18], or grown with chemical vapor deposition [19]. By carefully adjusting the parameters of the growth and etching process, different topologies can be achieved. Further requirements for a sufficiently good TCO are high transparency in the spectral domain between 350 and $1100 \mathrm{~nm}$, high conductivity and a surface on which the subsequent solar cell can be grown with marginal defects. Optimizing the fabrication is not straightforward, since some of these requirements contradict, e.g. the high free carrier density required for high conductivity lowers the transparency in the NIR due to free carrier absorption. Due to the complexity of this ongoing optimization process, various, deviating topologies are presently available and still under investigation. However, all these topologies perform below the Lambertian limit.

By means of rigorous simulations we will show here that by suitably scaling both the texture

\#146245 - \$15.00 USD Received 19 Apr 2011; revised 19 May 2011; accepted 23 May 2011; published 22 Jun 2011

(C) 2011 OSA

4 July 2011 / Vol. 19, No. S4 / OPTICS EXPRESS A867 
height and length, the cell performance approaches the Lambertian limit and may even exceed it in narrow spectral domains. Whether or not the Lambertian limit is approached is marginally affected by the actual topology. In fact, various textures could be pushed in their performance to reach this limit for an appropriate scaling. Moreover, the suitable scaling to reach the limit suggests smaller feature sizes as more optimal when compared to those already in place. Thus, a key message of this Letter is foremost a pleading for exploiting nanoscopic structures in photon management. Along this path from an optical point of view we disclose further aspects why certain textures perform better than others. It will be shown that they operate closer to the point of optimal scaling. It has to be stressed that our considerations focus only on the optical aspects of solar cells. Further issues related to the electrical properties might affect the overall performance as well and thus the ultimate choice of a certain texture.

\section{Numerical treatment}

(a)

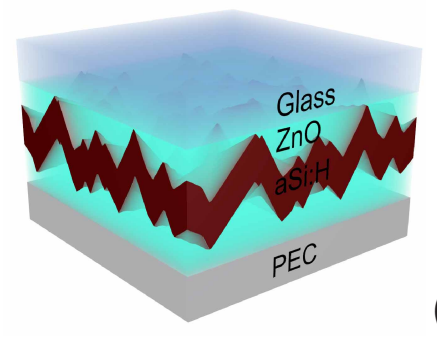

(b)
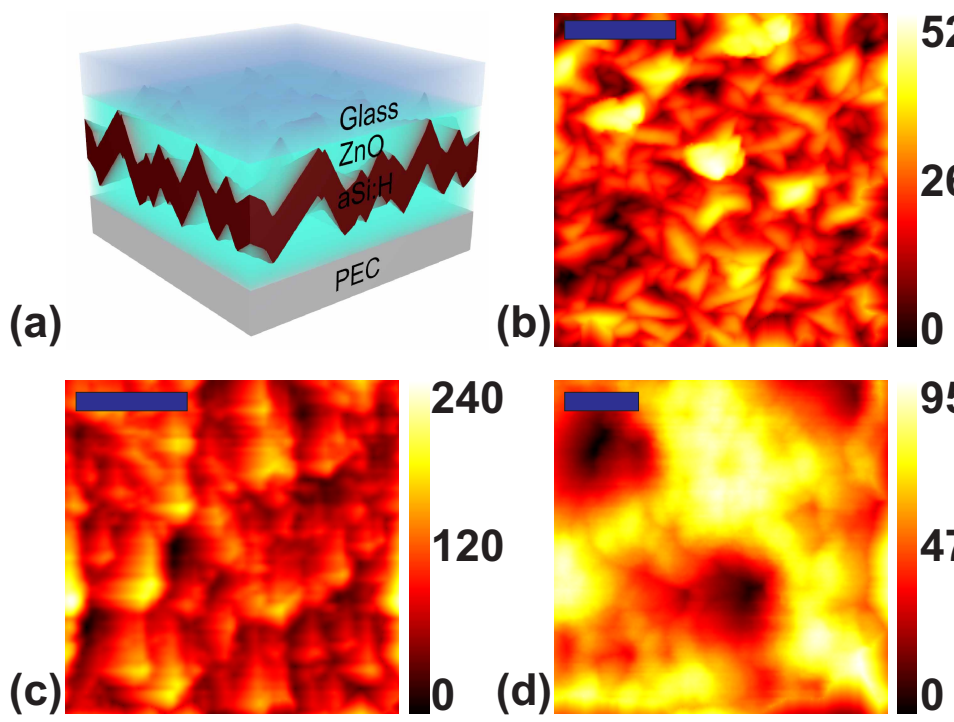

240

120

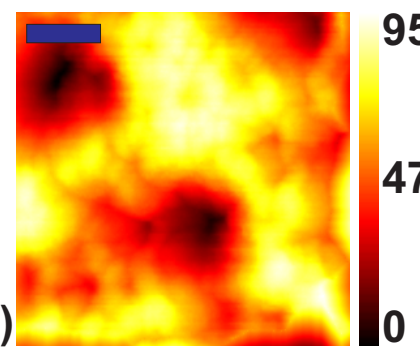

Fig. 1. (a) Three-dimensional schematic of the solar cell under consideration. A $250 \mathrm{~nm}$ thick aSi:H-layer is conformally deposited between textured $\mathrm{ZnO}$. The cell is finalized by a glass superstrate at the top and a perfect reflector at the bottom. Topographies in $\mathrm{nm}$ of the investigated textures correspond to superstrates fabricated in Neuchâtel (b), at Asahi (c) and in Jülich (d). The blue scale bars represent $1 \mu \mathrm{m}$.

An artistic view of the solar cell under consideration is shown in Fig. 1(a). The textured $\mathrm{ZnO}$ layer is deposited on a glass superstrate. In a following step a $250 \mathrm{~nm}$ thick aSi:H-layer is comformally deposited, which is followed by an additional $\mathrm{ZnO}$ layer. The cell is finalized by a planar perfect electric conductor (PEC) which has a minimum distance to the aSi:H layer of $100 \mathrm{~nm}$ and which provides complete back reflection.

We used the Rigorous Coupled Wave Analysis (RCWA) for the numerical treatment [20], which allows to solve Maxwell's equations rigorously for periodic structures in frequency domain. In our considerations we rely, therefore, on periodic super cells. The super cells were chosen sufficiently large to exclude any spurious influence. In RCWA the spatially varying dielectric properties of the material and all fields were expanded in a plane wave basis with $21 \times 21$ Fourier components, sufficient to assure convergence of all simulated quantities. For the illumination we assumed normally incident unpolarized light. For glass and $\mathrm{ZnO}$ we as- 
sumed perfect transparency and refractive indices of 1.5 and 2.0, respectively. The optical properties of aSi:H were taken from the literature and their precise spectral dependence was taken into account $[21,22]$. Due to the PEC at the backside, the spectrally resolved absorptance of the aSi:H layer $A(\lambda)$ is then given by $A(\lambda)=1-R(\lambda)$, with $R(\lambda)$ being the total reflectance of the investigated structure.

For the textures we relied on superstrates already discussed in literature [23]. Their topology [see Fig. 1(b)-1(d)] was recorded with an atomic force microscope. We considered the commercially available superstrate Asahi-U (characterized by lateral feature sizes in the range of $500 \mathrm{~nm}$ and a maximum texture height of $H_{\mathrm{tex}} \approx 250 \mathrm{~nm}$ ), superstrates fabricated in Neuchâtel [24] (lateral feature sizes in the range of $500 \mathrm{~nm}, H_{\text {tex }} \approx 500 \mathrm{~nm}$ ) and in Jülich [25] (lateral feature sizes in the range of $1-2 \mu \mathrm{m}, H_{\mathrm{tex}} \approx 1000 \mathrm{~nm}$ ). For the numerical implementation of these superstrates we relied on measured samples with dimensions of $3 \times 3 \mu \mathrm{m}^{2}$ for the Asahi-U and Neuchâtel textures, and on $4.5 \times 4.5 \mu \mathrm{m}^{2}$ for the Jülich superstrate. We modified these textures slightly to ensure that they are continuous at the boundaries if arranged in a periodic pattern. To evaluate the performance of the textures, we calculated the absorptance of the corresponding solar cell implemented as in Fig. 1(a) for 111 equally distributed wavelengths between 300 and $850 \mathrm{~nm}$.

\section{Impact of lateral scaling}

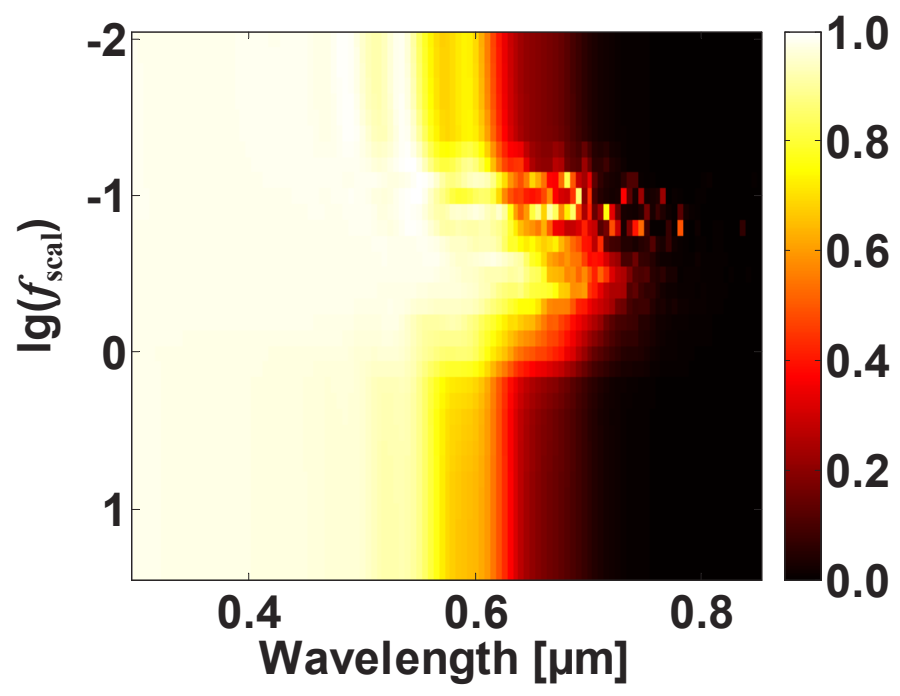

Fig. 2. Absorptance spectra of the considered solar cell for varying lateral scaling factors $f_{\text {scal }}$.

The calculated absorptance spectra for the Neuchâtel texture is shown in Fig. 2. In addition to the spectral dependency, we have varied the characteristic lateral length of the texture by a suitable scaling factor $f_{\text {scal }}$. To this end, we kept the height of the texture constant and calculated the absorptance for different lateral sizes of the unit cell given by $f_{\text {scal }}$. Similar graphs were obtained for the other superstrates. A scaling factor $f_{\text {scal }}$ of one $\left[\lg \left(f_{\text {scal }}\right)=0\right]$ corresponds to the texture as fabricated.

Prior to any further discussion, it has to be noted that light at wavelengths below $550 \mathrm{~nm}$ is completely absorbed once it enters the aSi:H-layer. The intrinsic absorption coefficient of aSi:H is sufficiently high that this is possible with the chosen material thickness. Hence, in this

\#146245 - \$15.00 USD Received 19 Apr 2011; revised 19 May 2011; accepted 23 May 2011; published 22 Jun 2011 (C) 2011 OSA

4 July 2011 / Vol. 19, No. S4 / OPTICS EXPRESS A869 
spectral domain the dominating loss mechanisms are reflections occurring at the glass/ZnOand at the $\mathrm{ZnO} / \mathrm{aSi}: \mathrm{H}$-interface. So, in general, the absorptance increase in this spectral domain can be directly attributed to the reduction of reflection losses by the texture . In the spectral domain between $550 \mathrm{~nm}$ and $800 \mathrm{~nm}$ the absorption coefficient of aSi:H drops and hence light trapping is needed in addition to effective light incoupling in order to realize a high absorptance. In our context this is the most relevant spectral domain for light trapping since such a shift of the spectral range of low absorptance towards longer wavelengths proves highly beneficial for increasing the photocurrent of the solar cell. At wavelengths exceeding $800 \mathrm{~nm}$ the absorptance is negligible regardless of any light trapping schemes; thus, this spectral domain does not need to be considered.

At first we want to discuss the absorptance for wavelengths below $550 \mathrm{~nm}$ in more detail. In the limiting case of very small lateral scaling factors $\left(f_{\text {scal }} \lesssim 10^{-2}\right)$ the impinging light cannot resolve the spatial features and does not undergo any scattering events. The texture is deep sub-wavelength and the illumination rather experiences an effective medium with a continuously varying refractive index. This is beneficial for reducing the reflection losses [26] and for keeping the absorption large. For intermediate scaling factors $\left(f_{\text {scal }} \lesssim 1\right)$ the impinging light can resolve the texture and is hence partially scattered. The light back scattered at the $\mathrm{ZnO} / \mathrm{aSi}: \mathrm{H}$-interface can then undergo an additional transmission event at the $\mathrm{ZnO} / \mathrm{aSi}: \mathrm{H}-$ interface. This might happen if it is directly reflected onto another crater face, or if it detours to the glass/ZnO-interface, where it experiences total internal reflection (TIR) [27]. As a consequence, both additional transmission events lead to the reduction of reflection losses. For very large lateral scaling factors $\left(f_{\text {scal }}>10\right)$ most features of the texture are larger than the impinging wavelengths and, consequently, the light hits a surface which is locally almost flat. Hence, the cell can be locally described as a slightly tilted planar layer stack. Therefore, in this limit the light will be reflected at all interfaces, especially at the glass/ZnO- and at the $\mathrm{ZnO} / \mathrm{aSi}$ :H-interface. These reflection losses will decrease the amount of light coupled into the aSi:H-layer and reduce the absorptance. Thus, we conclude, that in this limit the absorption will be comparably low. Following the discussion above, a gradual decrease of the absorptance in the spectral domain below $550 \mathrm{~nm}$ for increasing $f_{\text {scal }}$ is expected as a general trend, which is observed in more detail in Fig. 3 where the absorptance is shown for several wavelengths. Since the considered texture is of random nature, no particular wavelength should be generally preferred in the considered spectral range. Hence, it can be anticipated that the described tendency below $550 \mathrm{~nm}$ should likewise hold for wavelengths above $550 \mathrm{~nm}$. This is valid in the limiting cases of either very large or very small scaling factors, since textures in these limits do not scatter light but may reduce reflection losses only. However as can be also observed in Fig. 2 for wavelengths exceeding $550 \mathrm{~nm}$ a resonance domain exists in a certain interval of scaling factors $f_{\text {scal }}$, which are, interestingly enough, slightly smaller than unity $\left[\lg \left(f_{\text {scal }}\right)<0\right]$. For such textures with lateral features in the order of around $150 \mathrm{~nm}$, several effects may occur, which result in a stronger light localization within the aSi:H-layer. First of all, although the impinging light can resolve such large features the texture still benefits from reduced reflection losses as already discussed above. An additional effect promoting localization is the excitation of leaky modes within the aSi:H film. Note that due to the random nature of the considered texture, which is characterized by many different tangential wave vector components, guided modes are only weakly excited [28]. Another mechanism is the lightning rod effect, which can be associated with localization of light near points of strong curvature of the surface [29]. And at last we expect scattered light, which is trapped due to TIR at the glass/ZnO-interface and can undergo additional absorption events. All these effects which cause a localization translate directly into an increased local absorption since it is proportional to the local field intensity.

In a more physical picture, the structure can be understood as a strongly scattering element

\#146245 - \$15.00 USD Received 19 Apr 2011; revised 19 May 2011; accepted 23 May 2011; published 22 Jun 2011 (C) 2011 OSA

4 July 2011 / Vol. 19, No. S4 / OPTICS EXPRESS A870 


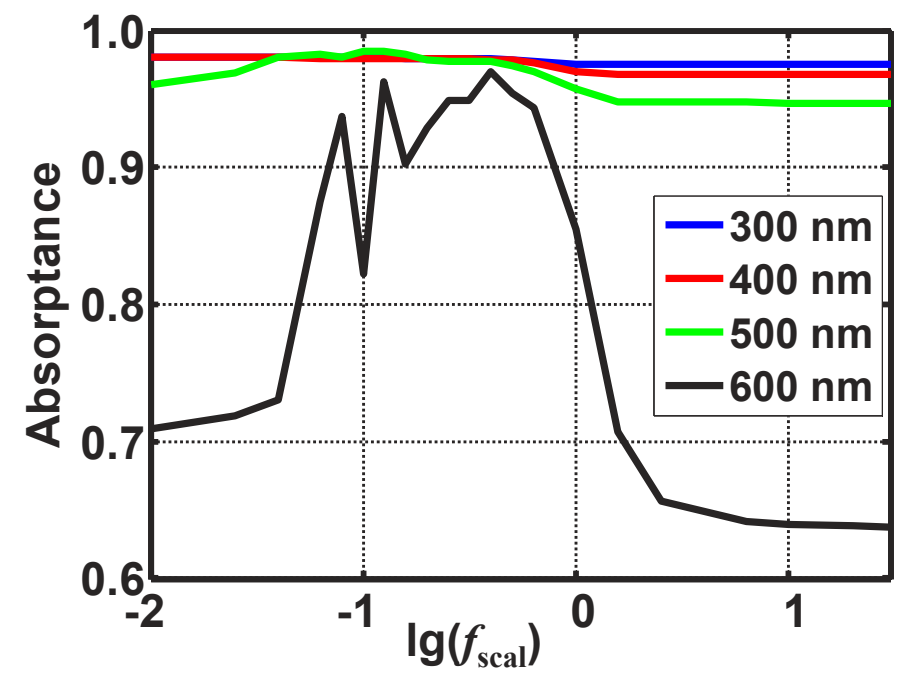

Fig. 3. Absorptance at several wavelengths versus the lateral scaling factor.

which supports a large number of diffraction channels. They are all excited with nonzero amplitudes where the exact excitation strength is difficult to determine due to the complexity of the structure. The absorptance in the limiting cases of either small or large scaling factors is much less whereas the performance of the solar cell in this intermediate resonance regime is largely enhanced. For scaled feature sizes of only a few tens of nanometers, the diffraction channels are not excited at all since light experiences effectively a homogenous medium. For structures much larger than the optimal scaling the structure appears almost flat and no significant amount of diffraction channels is excited, which would be required to approach the Lambertian limit [17]. Only in this resonance domain the structure exploits them and allows for a much larger absorptance than a solar cell operated in either of the limiting regimes. Furthermore, there are no symmetry considerations prohibiting the excitation of a significant amount of the diffraction channels [15] due to the randomness of the texture. Weighting the calculated absorptance with the number of incident photons given by the AM1.5g solar spectrum [30] and integrating over the spectral domain of interest provides the number of photons absorbed in the aSi:H layer. An upper limit of the short circuit current density $\left(J_{\mathrm{SC}}\right)$ can be obtained by assuming that each absorbed photon generates one electron-hole-pair and that all generated carriers are perfectly collected at the corresponding contacts. The $J_{\mathrm{SC}}$ calculated for the different textures is plotted in Fig. 4. The black horizontal line depicts the Lambertian limit, where the spectrally dependent absorptance of the aSi:H layer was calculated according to:

$$
A(\lambda)=\frac{0.98(1-\exp [-4 \alpha(\lambda) d])}{1-\left[1-1.5^{2} / n^{\prime}(\lambda)^{2}\right] \exp [-4 \alpha(\lambda) d]},
$$

which is applicable also in the case of a non-vanishing absorption [31]. Here, $\alpha(\lambda)$ is the spectrally resolved absorption coefficient given by $\alpha(\lambda)=4 \pi n^{\prime \prime}(\lambda) / \lambda, n^{\prime}(\lambda)$ and $n^{\prime \prime}(\lambda)$ are the real and imaginary part of the refractive index of aSi:H, respectively, and $d$ is the thickness of the aSi:H layer amounting to $250 \mathrm{~nm}$. The factor of 0.98 accounts to the transmission of the glass/ZnO-interface. The corresponding reflection losses can be further decreased by incorporating additional antireflection layers between glass and $\mathrm{ZnO}$, but that aspect is of minor importance for the present consideration. We distinguish in Fig. 4 the different superstrates as

\#146245 - \$15.00 USD Received 19 Apr 2011; revised 19 May 2011; accepted 23 May 2011; published 22 Jun 2011 (C) 2011 OSA 


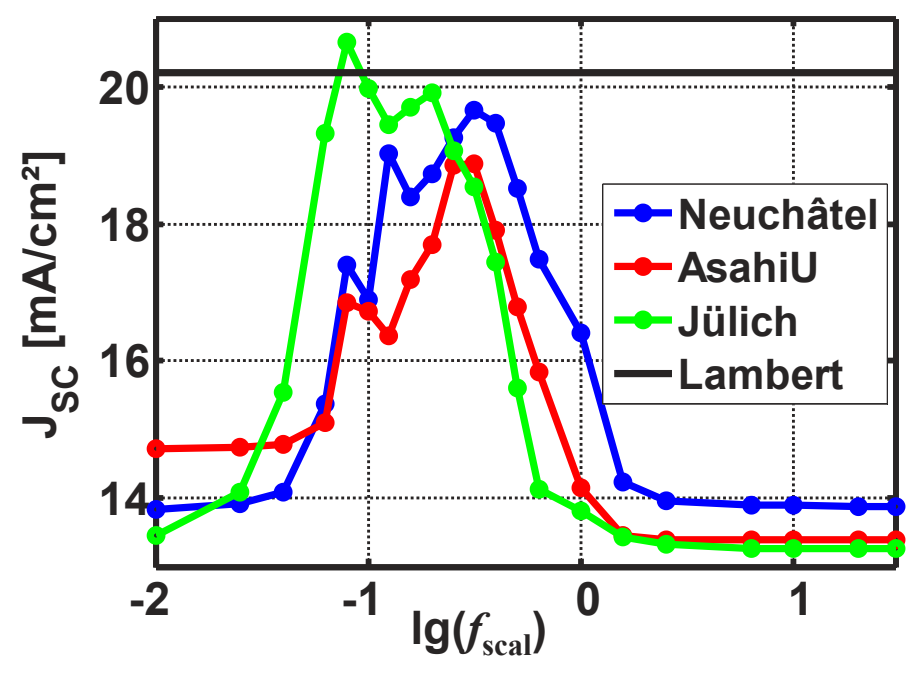

Fig. 4. Short circuit current density of the considered solar cell for varying lateral scaling factors $f_{\text {scal }}$. The black horizontal line depicts the Lambertian limit for a reflection loss of $2.0 \%$.

well as the dependency of the short circuit current density on the scaling factor. As can be observed, the general tendency of all curves coincides. Of course, due to the differing lateral feature sizes of the unscaled textures, these curves are slightly shifted with respect to each other, but otherwise the functional dependency is fully preserved. Note, that a scaling factor of unity $\left[\lg \left(f_{\text {scal }}\right)=0\right]$ is related to the superstrates as fabricated. For very small or very large $f_{\text {scal }}$, the $J_{\mathrm{SC}} \mathrm{S}$ are almost constant and determined by the texture-induced reduction of reflection losses, as already discussed. In the resonance domain defined above the increased absorptance around the absorption edge of aSi:H leads to an increased $J_{\mathrm{SC}}$. For small values of $f_{\text {scal }}$ only a small fraction of the texture features are already large enough to provide sufficient localization of the light within the aSi:H layer which explains the smooth increase of $J_{\mathrm{SC}}$ with increasing $f_{\text {scal }}$. Analogously for large values of $f_{\text {scal }}$, only a small fraction of the texture features is still sufficiently small to localize the light which explains the smooth decrease of $J_{\mathrm{SC}}$ with increasing $f_{\text {scal }}$. Since all shown curves have their maximum in the same range of $19-21 \mathrm{~mA} / \mathrm{cm}^{2}$, we conclude, that for achieving a high $J_{\mathrm{SC}}$ with randomly textured surfaces, the exact shape of the textures is of minor importance. A more severe tuning issue consists in adjusting the lateral feature size.

\section{Impact of vertical scaling}

To further back the conclusion that the exact texture shape is of minor importance, we calculated $J_{\mathrm{SC}}$ as a function of the maximum texture height $H_{\mathrm{tex}}$, i.e. we scaled all textures studied along the vertical dimension, where $f_{\text {scal }}$ was chosen from the maxima in Fig. 4. The results are shown in Fig. 5, where the large open circle in each curve corresponds to the texture height as measured. For small heights, the textures are almost flat and the absorptance suffers from high reflection losses. For increasing $H_{\text {tex }}$ more light is coupled in and the texture yield an additional light localization inside the aSi:H layer. However, for texture heights above $200 \mathrm{~nm}$, $J_{\mathrm{SC}}$ seems to saturate. In line with the argumentation above, the saturation is due to an optimum excitation of all diffractive channels by the structure. The question to be posed is, how closely

\#146245 - \$15.00 USD Received 19 Apr 2011; revised 19 May 2011; accepted 23 May 2011; published 22 Jun 2011 (C) 2011 OSA

4 July 2011 / Vol. 19, No. S4 / OPTICS EXPRESS A872 


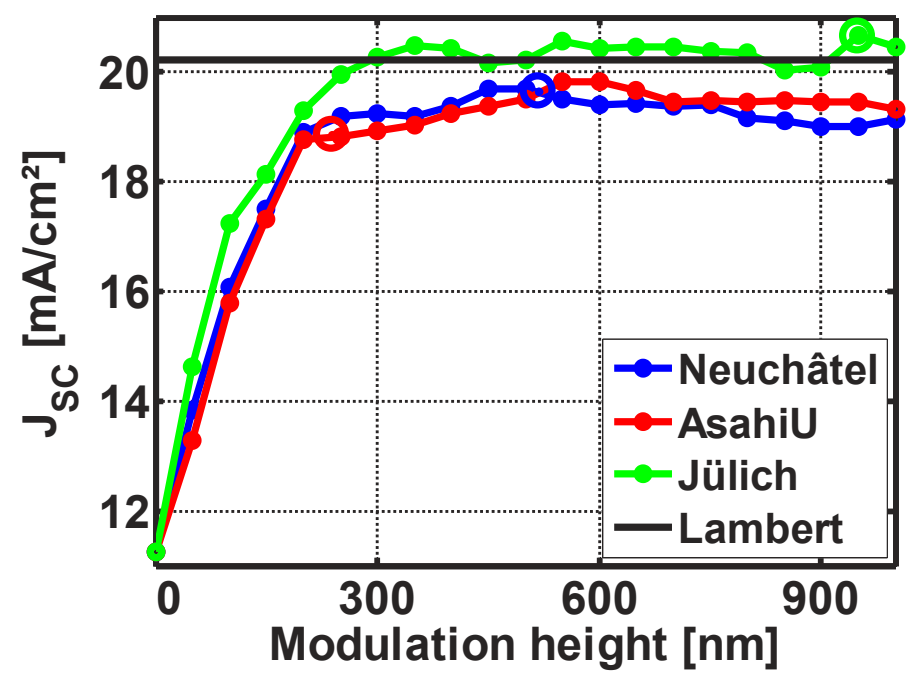

Fig. 5. Short circuit current density of the considered solar cell for a varying modulation height of the texture. The black horizontal line depicts the Lambertian limit for a reflection loss at the glass $/ \mathrm{ZnO}$-interface of $\approx 2.0 \%$.

this saturation limit approaches the Lambertian limit.

\section{Approaching the Lambertian limit}

Figure 6 visualizes the calculated absorptance spectra for the different superstrates corresponding to the maxima in Fig. 4. Note that the shown curves are smoothed, in order to make the plots more accessible to the eye. It can be observed, that all superstrates, except the quite shallow Asahi-U one, exhibit very low reflection losses for wavelengths below $550 \mathrm{~nm}$. For comparison the absorptance calculated for the Lambertian limit according to Eq. (1), is also shown as the black curve. The most important information which can be extracted from the figure is that the absorptance spectra for the most suitably scaled textures are all quite close to the Lambertian limit. Hence, it might be concluded that the incident light is perfectly scattered. Table 1 summarizes the obtained results quantitatively in terms of $J_{\mathrm{SC}}$. The data shown confirm that the optimized lateral feature size amounts to about $150 \mathrm{~nm}$ which is accessible by modern nanofabrication technologies [32]. Moreover, this feature size does not deviate much from those of already available textures. Their point of operation is yet simply chosen slightly inappropriate, though a small reduction of the lateral feature size towards the nanoscopic range will potentially yield a big leap towards a much more efficient solar cell. Moreover, the essential finding here is that the subtle topological details of the texture are of secondary importance only.

\section{Summary}

In conclusion, in this paper we have investigated the dependence of the absorptance of thin-film solar cells incorporating a randomly textured superstrate, when laterally scaling the texture. It has been shown that for sufficiently large modulation heights the short circuit current density strongly depends on the lateral feature size. For lateral feature sizes in the optimum range around $150 \mathrm{~nm}$, a further increase of the aspect ratio by increasing the modulation height leads only to minor changes in the $J_{\mathrm{SC}}$. Furthermore we could show, that such an optimized texture

\#146245 - \$15.00 USD Received 19 Apr 2011; revised 19 May 2011; accepted 23 May 2011; published 22 Jun 2011 (C) 2011 OSA 


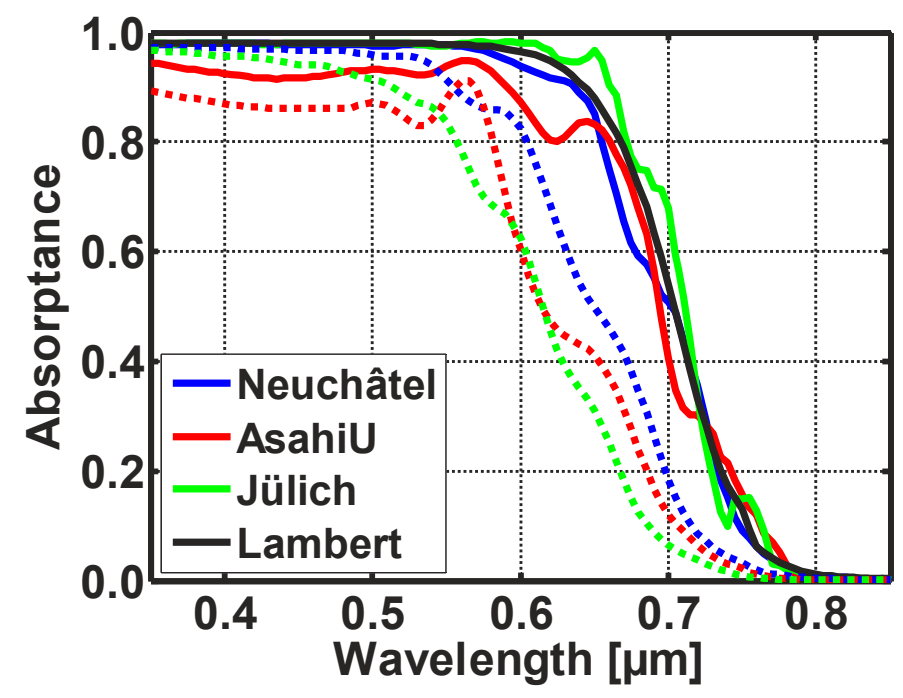

Fig. 6. Calculated absorptance spectra for optimized lateral scaling factors for the different superstrates. The black curve depicts the Lambertian limit assuming a reflection loss at the glass $/ \mathrm{ZnO}$-interface of $\approx 2.0 \%$. The dotted curves are calculated for the unscaled textures.

Table 1. Parameters of Optimized Textures Discussed in this Paper

\begin{tabular}{cccc}
\hline \hline Superstrate & $\lg \left(f_{\text {scal }}\right)$ & feature size $[\mathrm{nm}]$ & $J_{\mathrm{SC}}\left[\mathrm{mA} / \mathrm{cm}^{2}\right]$ \\
\hline Neuchâtel & -0.5 & 160 & 19.67 \\
Asahi-U & -0.5 & 160 & 18.87 \\
Jülich & -1.1 & 120 & 20.66 \\
Lambert & & & 20.22 \\
\hline \hline
\end{tabular}

leads to absorptances approaching the Lambertian limit. It may be anticipated that this work will facilitate the further optimization of textured superstrates utilized in thin-film photovoltaics.

\section{Acknowledgments}

We acknowledge the partial financial support of this work by the Deutsche Forschungsgemeinschaft (Nanosun (PAK 88)) and the Thüringer Aufbaubank (SolLux). Some computations utilized the IBM p690 cluster JUMP of the Forschungszentrum in Jülich, Germany. T. K. acknowledges support through an Imperial College Junior Research Fellowship. We would like to thank our colleagues in Jülich and Neuchâtel for providing us with the topology of their superstrates.

\#146245 - \$15.00 USD Received 19 Apr 2011; revised 19 May 2011; accepted 23 May 2011; published 22 Jun 2011 (C) 2011 OSA 